

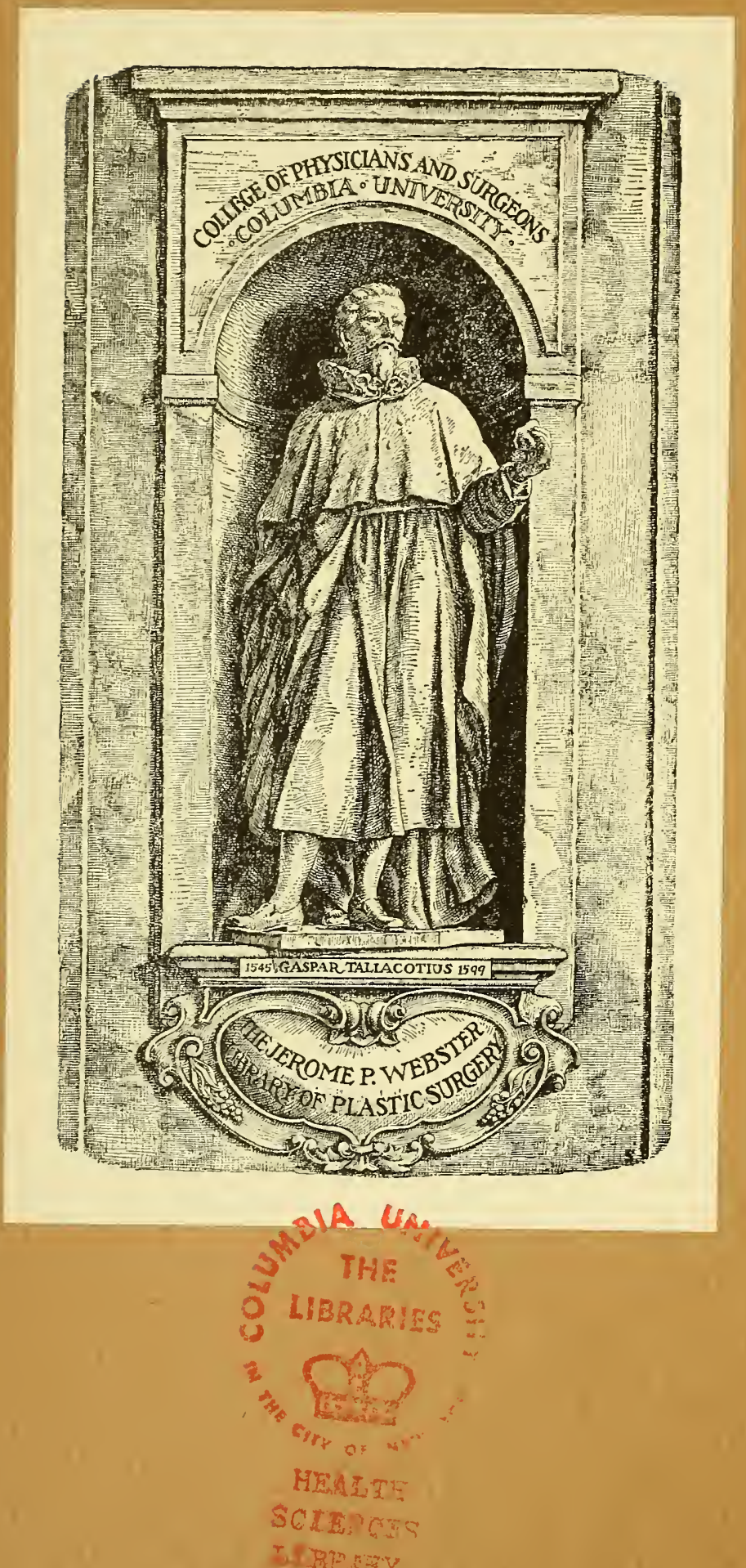


Digitized by the Internet Archive in 2010 with funding from Open Knowledge Commons 
Über plastische Deckung

von

Hautdefekten an den Extremitäten durch gestielte Hautlappen

aus entfernten Körperteilen.

\section{In augural-Dissertation}

$\operatorname{zin}$

Erlanǵungog der Doctorwürde in der Medicin und Chirurggie,

\section{welche}

mit Genehmigıng der hohen medicinischen Fakultät

der

vereinigten Friedrichs-Universität Halle-Wittenberg

zugleich mit den Thesen

Dienstag, den 15. August 1893, Vormittags II Uhr

öffentlich verteidigen wird

\section{Georg Hartung}

aus Königsberg i. Pr.

Referent: Herr Prof. Dr. von Bramann.

\section{Opponenten :}

Herr Dr. med. Willy Morro, Assistenzarzt.

Herr Karl Küstermann, cand. med.

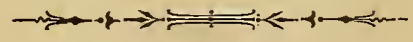

Halle a. S.,

Hof buchdruckerei von C. A. Ka emmerer \& Co. 1893. 


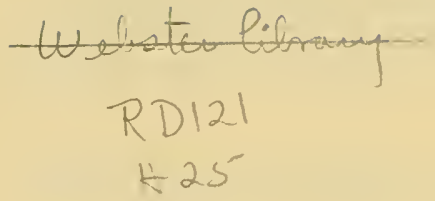

Imprimatur

Prof. Dr. von Bramann

b. t. Decanus 


\section{Seinen Eltern}

in Dankbarkeit

gewidmet.

6- (2) (3) 

Plastische Operationen, unter welchen man im allgemeinen die Deckung eines angebornen nder erworbenen Defekts versteht, wurden bekanntlich schon in Altertum von indischen Ärzten häufig ausgeführt, und zwar die Rhino-, Cheilo- und Otoplastik. Veranlassung und liänfige Gelegenheit dazu gab die noch jetzt im Morgenlande und besonders in Indien gebräuchliche Strafe, Nasen, Ohren u. s. w. abzilschneiden. Der Hantlappen, welcher zur Deckung des Defektes dienen sollte, wurde aus dessen unmittelbarer Nachbarschaft, aus der Haut der Wange gebildet.

Die ersten Angaben über plastische Operationen im Abendlande, welche wir bei Cels us ("medicinae libri octo"), Galen (2. Jahrh. n. Chr.) und Paulus von Aegina (7. Jahrh. n. (hr.) finden, sind äusserst dürftig und beziehen sich mehr auf seitliche Hautverschiebung als auf Hautüberpflanzung.

Erst im 15. Jahrhundert kamen die plastischen Operationen mehr zur Geltung, und zwar in Calabrien; es waren besonders der Wundarzt $\mathrm{Branca}$ aus Catania und sein Sohn Antonio, welche sich um die Vervollkommung dieser Kunst verdient machten. Branca benutzte zur Rhinoplastik einen gestielten Hautlappen aus der benachbarten Wangenhaut, sein Sohn entnahm das Hauptstück zum Ersatz des Nasendefekts dem Oberarm, also einer entfernten Körpergegend. Die Ausïbung der Plastik blieb jedoch lange Zunftgeheimnis der Calabrischen Ärzte, bis endlich durch Gasparo Tagliacozza, Professor der Anatomie und Medizin zu Bologna 
(1546-1599), sie zur allgemeinen Kenntnis gelangte. Er erreichte es aber nicht, die damaligen Chirurgen dauernd für plastische Operationen zu begeistern. Die Schwerfälligkeit seiner Darstellung, seine umständlichen Vorschriften und nicht zum Geringsten die Interessenlosigkeit der damaligen Ärzte an der Litteratur brachten es dahin, das ,eine der glänzendsten Bereicherungen der Chirurgie aufs neue für 2 Jahrhunderte. in Vergessenheit sank."

Auch waren die Beschwerden. vor allem die Schmerzen bei einer solchen Operation in jener Zeit, als man Anaesthetica nicht kannte, derartig, dass sich nur wenig Patienten dazı herbeiliessen, und die Ärzte selbst davon abrieten.

Seltsame Gerïchte über die neugebildeten Körperteile, Verspottungen und wohl auch zahlreiche Mlisserfolge brachten schliesslich das Kapitel von den plastischen Operationen gänzlich zum Stillschweigen, und erst dem 19. Jahrhundert war es vorbehalten, sie den Dunkel der Vergessenheit zu entreissen und, zum Heile der leidenden Menschheit, zu ungeahnter Vervollkommnung zu bringen.

Karl Ferdinand Gräfe (1816), welcher die Versuche mit plastischen Operationen eifrig wieder aufgenommen hatte, kürzte das umständliche Verfahren Tagliacozzas bedeutend ab und nannte seine Methode, im Gegensatze zur ,indischen" und ,,italienischen", die „deutsche."

Unter seinen Nachfolgern muss man hauptsächlich Dieffenbach's (1822) gedenken, welcher sich sehr grosse Verdienste um die Vervollkommung der Plastik erworben hat, und schon erfolgreiche Untersuchungen der physiologischen Vorgänge an transplantierten Hautlappen anstellte.

Man kann die plastische Kunst der früheren Zeit, bis zur Mitte des 19. Jahrhunderts, mit Recht eine ,ars decoratoria" nennen, nur kosmetische Rïcksichten forderten zu plastischen Operationen auf, und diese waren fast nur auf die Wiederherste!lung zerstörter Nasen, Ohren und Lippen beschränkt; kosmetische Störungen gaben also hauptsächlich die Indikation zur Plastik ab. 
Erst in der neueren Zeit hat man versucht, auch, wenn praktische Rücksichten es verlangen, plastisch zu operieren, also zu wahren Heilzwecken! Wenn man auch in jener Zeit von bedeutenden Erfolgen noch nicht reden konnte, so sind doch die Bemühungen sehr anzuerkennen, welche schon damals die Chirurgen sich gaben, durch ein Trauma, ein Geschwür und andere Ursachen veranlasste Hautdefekte auf plastischem Wege zur Heilung zu bringen.

Besonders an den Extremitäten sind ja derartige Substanzverluste in hohem Grade geeignet, bedeutende Funktionsstörungen hervorzurufen, und oft verfiel das betr., unbraulchbare Glied, allen Heilversuchen trotzend, unweigerlich der Amputation.

Der bedeutenden Beschwerden wegen, welche bei der Rhinoplastik durch die lange Aneinanderheftung des Arms an den Kopf bis zur Anheilung des überpflanzten Hautstiicks, dem Patienten erwuchsen, erstrebte man schon lange eine Abänderung. Als nun ausserdem Fälle vorkamen, bei denen weder die Haut des Gesichts noch die der Arme zum Ersatz gebraucht werden konnte, verfiel man wieder auf die sog. ältere indische Methode, gänzlich abgetrennte Hautstiicke zur plastischen Deckung zu benutzen.

So verpflanzte Bünger in Marburg ${ }^{1}$ ) bei einem solchen Patienten ein gänzlich abgetrenntes, dem Oberschenkel entlehntes Hautstïck auf einen Nasendefekt, wie er angiebt, mit teilweisem Erfolge.

Zahlreiche Chirurgen, wie Dzondi in Halle, Dieffenbach, Wutzer u. A., welche dann auch Versuche mit ganz abgelössten Hautlappen anstellten, hatten jedoch keinerlei Erfolg zu verzeichnen.

Man liess trotzdem in den Bestrebungen nicht nach, die Versuche wurden mit Eifer fortgesetzt, und im Jahre 1869 feierte die plastische Chirurgie durch Réverdins Entdeckung einen grossartigen Triumph. Ausgehend von dem durch die Erfahrung bekannten Naturgesetze, „, dass die

1) Bünger. Gr. u. W. Journ. 1823, Bd. 4 . Gelungener Versuch einer Nasenbildung aus einem völlig getrennten Hautstïcke aus dem Beine. 
Überhüutung einer granulierenden Wundfäche nur von der Hautgrenze her erfolgt, und dass zur Möglichkeit einer Vernarbung Cutisgewebe die conditio sine qua non bildet", versuchte Réverdin, ein junger französischer Schweizer Mediziner, kleine Stïckchen Haut, welche er andern Körperstellen entnahm, auf Granulationsgewebe ïberzupflanzen und sie dort zur Heilung zu bringen. Der Versuch gelang: zwei dem andern Oberarm entnoumene Hautstiickchen heilten auf einem traumatischen Defekt des Arms alı. Seine Meinung zwar, nur Epidermis überpflanzt zu hahen, erwies sich als irrig. Deprès wies ihm nach, dass es Cutis in ganzer Dicke gewesen war. Jedoch gab man die Versuche, Cutisläppchen mit Unterhautzellgewebe zu verpflanzen, balı wieder auf, da trotz mannigfacher Modifikationen und Abänderungen bei der Operation der Erfolg ausblieb. Nach Réverdin sollten die Läppchen nur aus Epidermis und Corium bestehen und Erbsengrösse nicht iiberschreiten. Der Defekt musste eine gut aussehende Granulationsfläche darstellen. Entnommen wurden die Läppchen dem Patienten selbst als auch andern Individuen, dann auch frisch amputierten Gliedern und Tieren.

Längere Zeit vor Ré ve r'd in batte sich schon Thier'sch eifrigst, aber erfolglos, mit Transplantationen versucht. Durch Réverdins Erfolge wurde sein Interesse noch gesteigert, und schon im Jahre 1874 konnte er neue, wichtige Beobachtungen über die Anheilung von Haut auf Granulationsgewebe mitteilen $\mathbf{1}$ ).

Auf Grund seiner mikroskopischen Untersuchungen kam er darauf, die oberflächliche Schicht der. Granulationen, mit den senkrecht aufsteigenden Gefässschlingen vor Auflegen der Läppchen erst abzutragen, sodass , die untere, aus straffem Bindegewebe bestehende Schicht mit ihrem horizontal gelagerten Gefässnetze" freigelegt wurde.

1) Thiersch. Leipzig 1874. Über die feineren anatomischen Veränderungen bei Aufheilung von Haut auf Granulationen. 
Im Jahre 1886 veröffentlichte er eine neue Transplantationsmethode, welche sich von der Réverdin's hauptsächlich durch folgendes unterschied:

Während wie bereits erwähnt, Réverdin die Grösse des zu überpflanzenden Hautstïckes bis erbsengross angab, bediente sich Thiersch mit Erfolg bedeutend grösserer Hautläppchen von $10-14 \mathrm{~cm}$ Länge und $2-3 \mathrm{~cm}$ Breite. Durch Abschaben der oberflächlichen Granulationsschicht mit einem Rasiermesser stellte er eine frische Wundfläche her worauf er, nach sorgfältigster Blutstillung die Läppchen auflegte. Diese bestanden aus Epidermis, dem Papillarkörper, mit dem rete Malpighi und einer Lage glatten Stromas (=Corium). Die Thiersch'schen Läppchen hatten den Vorzug, grösser und bedentend schmiegsamer zu sein und leichter anzuheilen.

Die zahlreichen Versuche, welche daraufhin mit Transplantationen nach $R$ éverdin und besonders nach Thiersch angestellt wurden, übertrafen zuerst alle Erwartungen. Durch die zahllosen glücklichen Erfolge konnten sich die Indikationen zur Deckung von Haut-, Weichteil- und Knochendefekten durch Transplantationen immer mehr erweitern. Nach Verletzungen, nach Operationen (Entfernung von Geschwülsten), nach die Haut zerstörenden Krankheiten u. A. m. ergaben die neuen Methoden vorziigliche Resultate, sodass die Transplantationen bald ein Gemeingut aller Chirurgen wurden.

Am meisten wol forderten zur Operation auf ausgedehnte Hautnarben, Contrakturen an den Gelenken, entstanden nach Verbrenuungen, Verätzungen, Erfrierungen, Phlegmonen u. a., wodurch die Beweglichkeit der Glieder in hohem Grade beeinträchtigt oder völlig gehemmt, die Erwerbsfähigkeit der Patienten erheblich herabgesetzt oder ganz aufgehoben war.

Aber - trotz der zahlreichen glïcklichen Transplantationen, welche berichtet wurden, (O. Nage $\left.{ }^{1}\right)$ und $\mathrm{H}$ ii b scher $\left.{ }^{2}\right)$

1) 0. Nagel. Über die Erfolge der Hauttranspl. nach Thiersch. Aus der Tüb. chir. Klinik des Prof. Bruns. (Beitr. z. klin. Chir. 1889).

2 Ur. Hïbscher. Beiträge zur Hautverpflanzung nach Thiersch. Aus der baseler Chir. Klinik des Prof. Socin. (Beitr. z. klin. Chir, 1889). 
teilen mit, dass selbst Hautstreifen in der Länge von $30 \mathrm{~cm}$ anheilten), so liessen doch auch die Misserfolge nicht auf sich warten.

Diese Transplantationen waren, besonders bei ausgedehnten Hautdefekten, da nie alle verpflanzten Läppchen auf einmal anheilten, und deshalb zahlreiche Sitzungen nötig waren, recht umstündlich und zeitraubend. Die Körperstellen zwar, welchen man die Hautläppchen entnahm, zeigten schliesslich kaum sichtbare Narben.

Aber auch direkte Misserfolge traten ein:

An denjenigen Stellen des Körpers (besonders an den Extremitäten), welche äusseren Schädlichkeiten hauptsächlich ausgesetzt sind, an denen unmittelbar unter der Haut der Knochen liegt (z. B. Tibiakante, Ellenbogen), erzielten die Transplantationen keine dauernde Deckung der SubstanzverIuste. Die Läppchen heilten wol an, fielen aber bald, bei Einwirkung äusserer Schädlichkeiten, bei fortgesetztem Druck, Reibung u. s. w. wieder ab; oder es bildeten sich Einrisse und Schrunden, welche schwer verheilten. Ebenso erfolglos blieben die Versuche, tiefergreifende, z. B. nach langdanernden Phlegmonen entstandene Defekte in der vola manus, in der planta pedis zu ersetzen. Die neue, dünne Epidermisschicht kam direkt auf die durch das Tramma oder die Eiterung fieigelegten Sehnenscheiden, Gefässe und Nerven zu liegen und hielt selbst geringen Anforderungen nicht Stand. Stets blieben diese Stellen ein locus minoris resistentiae, beim Zufassen, beim Auftreten gingen die Hautläppchen bald zu Grunde.

Vorwiegend aber waren es geschwïrige Prozesse und besonders die beriichtigten Unterschenkelgeschwüre, welche hartnäckig der versuchten Überhäutung, durchTransplantationen Widerstand entgegensetzten.

Schon die grosse Anzahl der angegebenen Heilmethoden diesen Geschwüren gegenüber, beweist ihre Bösartigkeit. So wenig wie die seitlichen Incisionen (angegeben von John Simon und C. O. Weber 1865), die Circumcisionen nach 
Nussbaum, die mechanische Verzìehung der Haut durch extendierende Heftpflasterverbände (nach Maas und Schede), von den zahllosen "Vernarbungssalben" ganz abzusehen, ebenso wenig Erfolg erzielten hier Transplantationsversuche. An einzelnen Stellen heilten zwar die Läppchen an, die neugebildeten Narben brachen aber äusserst leicht wieder beim Gebrauch der Extremität anf; vor Recidiven war man nie sicher, und die erfolglosen Überhäutungsversuche konnteı in vielen Fällen das ultimum refugium, die Amputation des unbrauchbaren Gliedes, doch nicht verhüten.

In allen diesen, eben angegebenen Fällen, in welchen selbst durch T'hiersch'sche Transplantationen eine Überlaäutung und Vernarbung auf die Dauer unerreichbar ist, ist nun die Deckung des Substanzverlustes durch grosse, gestielte Hautlappen, welche Cutis in ganzer Dicke mit dem Unterhautzell - und Fettgewebe enthalten, allein wirksam, und diese Plastik hat sich seit Jahren durch ausgezeichnete Erfolge vielfach bewährt.

Meist handelt es sich um Defecte an den Extremitäten, deren freie Funktion dadurch behindert wird. Es sind also praktische Rücksichten, aus welchen plastische Operationen all 50 afficirten Extremitäten ausgeführt werden.

Zunächst bediente man sich zur plastischen Deckuug der Haut der Nachbarschaft; nur wenn diese nicht benutzt werden konnte, sei es, dass sie selbst durch narbige Schrumpfung verändert war, oder ihre Entfernung zu Funktionsstörungen Veranlassung geben würde, oder aber der zu deckende Defekt von zu grosser Ausdehnung war, entnahm man die Hautlappen entlegenen Körperstellen, natürlich nur solchen, welche eine Annäherung gestatteten.

Während man für Gesichtsdefekte seit langer Zeit schon frische, gestielte Lappen aus der Nachbarschaft, aus der Haut des Gesichtes, des Halses und des Kopfes verwandt hatte, um eine möglichst genaue Anpassung zu erzielen, hielt man es bei der Hernahme der Haut aus entfernten Körperteilen für ratsam, granulierende Lappen zu benutzen. Die 
Hautlappen wurden derart von ihrer Unterlage abgelöst, dass sie mit ihr nur noch durch 2 Stiele zusammenhingen; durch Einlegen ron Staniol oder dünnen Bleiplatten (Graefe) wurden die $W$ undflächen von einauder gehalten, eine gutartige Eiterung und Granulationsbildung erzielt. Dann erst wurde, nach Durchtrenmung eines Stieles der nummehr granulierende Lappen auf dem gleichfalls mit Granulationen bedeckten Defekt befestigt. Die Verwendung granulierender Lappen hielt nan für vorteilhafter, da sie durch die Granulationen schädlichen Einflïssen (zersetztem Urin bei Operation der ectopia vesicae, inficierendem $W$ undsekret u. a.) nicht so ausgesetzt sind, wie eine frische Wundfläche, und da die Sekretion vor der gefürchteten Austrocknungsnekrose, welche so oft die frischen Lappen zum Absterben bringen sollte, wie man glaulte, genügend schützte. Czerny meinte, granulierende Lappen, deren Gefässe in lebhafter Neubildung begriffen sind, könnten sich schneller mit dem neuen Nährboden in Verbindung setzen und wïrden deshalb schneller anheilen.

Die Nachteile erschienen allerdings auch nicht gering; die relative Dicke granulierender Lappen begünstigte ihre Aufwulstung und ein starkes Einkrempeln der Ränder, ihre Elastizität war im Vergleich mit frischen Lappen viel geringer.

Billroth (und Gussenbatier) gelang es 1874, durch Überpflanzen eines granulierenden Lappens mit den Dimensionen $11: 5,4 \mathrm{~cm}$, entnommen der Wade des anderen Beines, ein prominierendes ulcus der Ferse zur Heilung zu bringen. Gleichen Erfolg hatte Czerny 1876 und 1878 bei varicösen Unterschenkelgeschwüren, Thiersch bei Operation einer ectopia vesicae, Billroth bei Schluss einer Magenbauchwandfistel und einer Scheidenplastik.

Im Jahre 1818 hatte bereits Graefe empfohlen, zur Rhinoplastik einen frischen, gestielten Hautlappen dem Oberar'm zu entnehmen und sofort auf dem Defekte zu befestigen. Diese, die "dentsche" Methode, kürzte bedeutend das Verfahren 'Tagliacozza's ab, die langdauernden, beschwerlichen Vorbereitungen, den Lappen granulierend zu machen, blieben dem Patienten erspart. 
Zunächst scheinen jedoch die Erfolge mit der Verwendung frischer Hautlapnen nicht sehr zahlreich gewesen zu sein; auch $G r a e f e$ berichtet nur von einem erfolgreich verlaufenen Falle. Einen grossen T'eil der Schuld an diesen Misserfolgen muss man wol der recht mangelhaften Antisepsis jener Zeit zuschreiben.

Fabrici gelang es 1843 zuerst, einen frischen, gestielteu Hautlappen rom Thorax auf die Innenfläche der Hand zu verpflanzen.

Barwell entnahm 1866 mit Erfolg einen Hautlappen der seitlichen Hüfte, zur Beseitigung von Syndactilie, ebenso berichten Adams (1881), Socin (1883), Salzer (1886) und Czerny von guten Erfolgen.

Andere hingegen, wie Szigmondi in Wien und Wutzer, hatten mit der Plastik frischer Lappen wieder weniger Glück, letzterer schob das Misslingen der Operation allerdings auf die ungenügende Befestigung der beiden Extremitäten an einander.

M a a s in Würzburg beschäftigte sich sehr eingehend mit plastischen Operationsversuchen und war auch der erste, welcher die gelungene Überpflanzung eines frischen, gesticlteu Lappens von einem Unterschenkel auf den andern mitteilen konnte.

In seiner bekannten, ersten Arbeit ${ }^{1}$ ) zeigte er an der Hand von fünf Fällen, dass grosse, frische, gestielte Hautlappen, bei Beobachtung gewisser Vorsichtsmassregeln gut ernährt werden, eine widerstandsfähige Decke für den Defekt abgeben und nicht schrumpfen; ihre Sensibilität stellte sich nach einiger Zeit vollkommen wieder her.

Anf Grund seiner Versuche und Beobachtungen gab er eine Reihe von Regeln für diese Operationen an.

Zahlreiche Chirurgen, welche daraufhin nach seiner Methode operierten und sich im Allgemeinen an seine Regeln hielten, kunnten vorzägliche Resultate mitteilen. Ich erwähne

1) Maas Über Plastik mif frischen, gestielten Lappen aus entfernten Körperteilen. (Archiv f. klin. Chirurgie v. Laugenbeck Jahrg. 1855. Bd. 31). 
nur die von Nicoladoni (Innsbruck), Salzer jun. ${ }^{1}$ ), C. J üngst²) Francke ${ }^{3}$ ), von Eiselsberg.4), Wagner $\left.{ }^{5}\right)$, Czerny und Sawickib) mitgeteilten Erfolge.

II a s selbst teilte auf dem Chirurgen-Congress 1885 noch weitere Fälle ${ }^{7}$ ) von gelungener Plastik mit, desgleichen andere im Jahre $1886^{8}$ ).

Meine Aufgabe soll es nun sein, die Verwendung grosser, gestielter Hautlappen, entfernten Körperteilen entnomnell, zur Deckung von ausgedehnten Haut- und Weichteildefekten all den Extremitäten, zu belıandeln, und 7war mit besonderer Beriicksichtigung der Operations-Methode, wie sie in der chir. Klinik zu Halle des Herrn Prof. v. Bramann, welcher mir guitigst 5 neue Fälle zur Veröffentlichung zur Verfügung gestellt hat, iiblich ist.

Auch hier soll von Operationen aus kosmetischen Gründen nicht die Rede sein, sondern in erster Hinsicht von der Heilung derjenigen Substanzverluste an den Extremitäten, welche Ernährungs- und Funktionsstörungen veranlassen.

Der Zweck dieser Operationen ist also, unbrauchbare Glieder zu erhalten und brauchbar zu gestalten, und dadurch die Erwerbsfäligkeit der Patieuten wesentlich aufzubessern oder wiederherzustellen.

Die Indikationen für solche plastische Operationen an den Extremitäten sind zwar im Allgemeinen schon uben

1) Wiell. med. Wochenschrift 1887. No. 3 und 4.

2) Deutsche med. Woehenschr. 1887. No. 43.

3) Deutsche med. Wochenschr. 1889.

4) ebendas

5) Langenbecks Archiv XXXVI S. 381 und Verhandl. d. deutsch. Ges. f. Chir. 1887. I. S. 109.

6) Br. Sawicki. Üb. Transpl. gestielter Lappen aus entleg. Körperteilen. [gaceta lekarska 1890 No. 46-49] Centralblatt f. Chir. 1891.

7) IIaas. Autoplastik bei Geschwïren.

Centralblatt f. Chir. 1885. No. 24. Beilage.

8) Mlaas. Weitere Fälle v. Plastik mit frischen gesticlten Lappen aus entfernten Körperteilen.

Arehir f. klin. Chir. XXXIII. 1886. 
angedeutet, der Übersicht halber aber ist es erforderlich, dieselben hier nochmals zusammenzustellen:

Man kann die Hautdefekte einteilen in frische, granulierende und narbige.

Eine plastische Deckung durch grosse gestielte Hautlappen ist bei frischen und granulierenden Substanzverlusten indiciert, wenn dieselben so ausgedehnt oder so in die Tiefe greifend sind, dass anf eine andere Weise eine Heilung nicht zu Stande kommen kann.

Solche ausgedehnte Hautdefekte können entstehen durch Verletzungen aller Art, besonders durch Maschinengewalt, durch Verbrennungen, tiefgreifende phlegmonöse Prozesse, Operationen, durch die Haut zerstörende Krankheiten und vor allenı durch chronische Geschwüre.

Von besonderer Bedeutung sind diese Hantdefekte an denjenigen Körperstellen, hauptsächlich an den Extremitäten, welche äusseren Schädlichkeiten in höherem Grade ansgesetzt sind, z. B. vola manus, planta pedis, Kante der Tibia.

An den Gelenken kann man durch eine plastische Deckung bestehender Substanzverluste das Entstehen voll Narben und Contrakturen, und damit von Funktionsstörungen verhüten. Sind narbige Defekte bereits vorhanden, so lässt sich auch durch Excision der Narben und nachfolgende Plastik die Funktion des betr. Gelenkes wiederherscellen.

Von grosser Wichtigkeit bei einer plastischen Operation ist der Ort, von wo man den zu transplantierenden Lappen hernimmt. Zunächst ist hier wohl die Frage am Platze, ob es ratsam oder überhaupt zulässig ist, die Haut eines anderen Individuums als des Patienten selbst zur Verpflanzung zu benutzen.

Eine „Heteroplastik“ war schon Tagliacozza licht unbekannt. Ob schon zu jener Zeit viele Operationen auf diese Weise ausgefülırt worden sind, lässt sich durch die Literatur schlecht nachweisen.

Ein Punkt besonders war es, welcher eine Heteroplastik zu empfehlen schien: Wie Fischer bcobachtete, heilten 
Hautstiickchen besser an, wenu der gebende und der enp) fangende Ort anämisch gemacht war. Man versuclıte daher die Implantation von Haut frischer Leiclsen oder friscl amputierter Glieder und wendete zugleich bei den Patienten Blutleere an.

Für die Heteroplastik sprach auch die Beobachtung: dass bei alten, kachektischen Patienten ihnen selbst entnommene Hant nicht zur Anheilung gelangte, wohl aber dic anderer, jüngerer Individuen.

Von einigen Autoren wurden jedoch auch entgegengesetzte Resultate mitgeteilt.

Bald zeigte sich aber, welchen Gefahren man durcl eine Heteroplastik den Patienten gegebenen Falls aussetzte

Völcker ${ }^{1}$ ) teilt einen Fall mit, bei welchem durcl Hautverpflanzung Variola wälırend des Prodromalstadiums iibertragen wurde und der exitus letalis eintrat.

Unter andern berichtet auch $\mathrm{Czerny} \mathrm{y}^{2}$ ) iiber 2 Fälle, be: denen mit grosser Wahrscheinlichkeit T'uberkulose durcl Implantation von Hautstreifen übertragen worden ist, dies waren Gliedern, welche wegen Caries amputiert worden waren entnommen, wenn auch weit vom Erkrankungsherde entfernt

Wie Deubel ${ }^{3}$ ) angiebt, wurde 1881 durch transplantiert Hautstücke vom Sohne alf den Vater Syphilis verpflanzt Die Läppchen zerfielen unter Geschwürsbildung, und ers durch eine spezifische Kur gelangte die geschwürige Fläch allmählich zur Heilung.

Wemn schon diese Fälle beweisen, dass auch durcl Übertragung von Haut anscheinend gesunder Individuen ein Infektion möglich, eine Heteroplastik also zu verwerfen ist so wird man ansserden wohl kaum daran denken, bei eine Plastik mittels grosser gestielter Lappen die Haut eine ander'n Menschen zu benutzen.

1) Deutsche militärärztl. Zeitschrift 1872. Heft I

2) Centralblatt f. Chir. 1886.

(Beilage-Bericht über den Chir. Congress).

3) Gaz. méd, de Paris 1881 No. 55.

"Inoeulation de la syphilis par l'intermédiaric de greffes". 
$\mathrm{Da}$ in solchen Fällen Geber und Empfänger bis zur nheilung des Lappens an einander fixiert bleiben miissen, lässt schon die unangenehme und für beide Teile qualvolle tuation den Gedanken an eine Heteroplastik nicht anfommen.

In Kürze seien auch hier die Versuche erwähnt, durch berpflanzen von Tierhaut Hautdefekte beim Menschen zur eilung zu bringen. Barat toux und D ubonsquet-Laborderie achten den Vorschlag, die Bauchhaut von Fröschen, in Daumenagelgrossen Stückchen zı benutzen, und teilten Fälle mit, denen nach 4 Wochen Heilung eingetreten sein soll. Auch eorge R. Fowler beobachtete bei einer Negerin ein gutes esultat durch Überpflanzen von Froschhant; Heilung nach Monaten.

Phelps und M. E. van Meter verwandten die Haut inger, wenige Tage alter Hunde, P. Redard die von Hühnern, Ir Defektdeckung auf der menschlichen Kopffläche; mit roschhaut hatte er keine Erfolge erzielt.

Versuche mit Tierhaut laben sich nicht bewährt und nd nicht zu empfehlen.

Man wird daher, um wieder auf den Ort der Lappenahl zurückzukommen, in allen Fällen zunächst die Haut r Nachbarschaft, weil am bequemsten, zum Ersatz heranziehen suchen. Falls diese, z. B. durch narbige Schrumpfung erzu unbrauchbar sein sollte, erst dann wird man den autlappen anderen Körperteilen entnehmen.

Auch in den Fällen, welche hier in Betracht kommen, nd meistens die Defekte von solcher Ausdehnung, dass sie Is der Haut der Nachbarschaft nicht gedeckt werden innen; man wird sich also hier durchweg der Lappenbildung is entfernten Körperteilen zu bedienen haben, selbstverändlich, wenn eine Annäherung der betr. Teile überhaupt öglich ist.

Schon Tagliacozza hat die Frage erörtert, welche autgegenden als Ersatzmaterial bei plastischen Operationen n geeignetsten erscheinen; er empfahl die Armlaut in der icepsgegend als die zweckmässigste. 
Tie v. Langenbeck in seinen Vorlesungen über "Akiurgie" sich äussert, ist die Haut der Körperoberfläche keineswegs in gleichem Masse geeignet, das Material zum Wiederersatz zerstümmelter Teile zu geben. Die sehr verschiedene Dicke, die an manchen Körperstellen gar nicht, an andern als sehr dickes Polster vorhandene Fettschiclit unter der Haut, die Anheftung der Cutis an den darunter liegenden Teilen, die Gegenwart oder das Fehlen einer derben Fascie unter derselben, der Zusammenhang von Muskeln .mit der Cutis, endlich die Verteilung der Blutgefüsse in der Haut, alle diese Momente sind unstreitig von dem grössten Einfluss für das mehr oder weniger vollkommene Gelingen plastischer Operationen.

Für Defekte an der oberen Extremität und besonders am Vorderarme, wo ja hauptsächlich grössere Substanzverluste durch Verletzungen entstehen, erscheint zum Ersatz am geeignetsten die Haut des T'horax an der Seite in der Gegend des Rippenbogens. Hier ist eine bequeme Fixation der Extremität ermöglicht, die Haut daselbst ist sehr beweglich und elleichtert daher auch eine spätere Deckung des gesetzten Defekts ausserordentlich. B a r w e 11 empfahl zum Ersatz die seitliche Hüftgegend, Socin den Oberschenkel, Salzer benutzte, um eine zu starke Drehung des Hautlappenstiels zu vermeiden, für Defekte an der vola manus die Haut des Rückens, für das dorsum der Hand die seitliche Brusthaut. Die Haut des Rückens zu verwenden, dürfte sich deshalb nicht empfehlen, da es dann für den Patienten unmöglich oder jedenfalls nicht ratsam ist, im Bette die bequemste Lage, die Rückenlage einzunehmen.

In den meisten Fällen wird man, auch ohne die Basis des Lappens einer zu starken Drehung aussetzen zu müssen, mit der Hant der seitlichen Thoraxwand bequem auskommen. Bei weiblichen Patienten wird man gut thun, die Gegend der Schnürstelle del Kleider für die Narbe zu vermeiden.

Für Defekte an der unteren Extremität gilt folgendes: Für den Oberschenkel eignet sich sowolıl die Haut des 
andern Ober- wie Unterschenkels zum Frsatz. Sitzt der Defekt auf einem Unterschenkel, so benutzt man die Haut des andern Ober- oder Unterschenkels.

In allen diesen Eällen ist eine relativ bequeme Fixation beider Extremitäten möglich. $\mathrm{P}_{0}$ ncet $^{1}$ ) Jagerte bei einem Defekt, welclier das untere äussere Viertel des Unterschenkcls betraf, den Fuss an dieselbe Gesässseite an. Diese Fixation wird jedoch wol niclit bei jedem Patienten gelingen.

Es ist wol kaum nötig, besonders darauf hinzuweisen, dass auch dicsen Operationen cine sorgfältige, antiseptische Reinigung der Körpcrstelle, welcher der Lappen entnommen werden soll, voranzugehen hat. Die Operation selbst muss unter streng antiseptischen oder besser aseptischen Cautelen verlaufen.

Selbstverständlich ist bei jeder plastischen Operation von diesem Umfange Narkose unerlässlich.

Die Frage, ob man die betr. Körperteile (in Betracht kommen natürlich nur die Extremitäten) vor der Operation anämisch machen soll, hat die Chirurgen lange Zeit beschäftigt. Durch vielfache Versuche zeigte sich, dass bei diesen Operationen Esmarchsche Blutleere, die künstliche Anämie durch elastischen Bindendruck, im Allgemeinen nicht anzuraten ist.

Mit senkrechter Elevation des betr. Gliedes vor der Operation wird man völlig auskommen. Anderfalls kann eine Nachblutung aus den durchschnittenen Gefässen des Hautlappens den ganzen Erfolg in Frage setzell.

Was die Grösse des zu bildenden Lappens an betrifft, so muss dieselbe der Ausdehnung des Substanzverlustes entsprechen, sodassder Lappen den Defekt ohne Spannung ausfüllen kann. Früher glaubte man, dass, je grösser der Lappen sei, um so schwieriger die Anheilung zu stande kommen könne.

1) P o n c e t. Sur deux observations d'Autoplastic, pas la methode italienne modifiée. (Bull. et mêm. de la soc. de chir. de Paris T. XIV. p. 29). 
Zahlreiche Fälle aus der Literatur beweisen aber, dass die Grösse des Lappens ziemlich gleichgïltig für die Anheilung ist, vorausgesetzt, dass seine Basis eine entsprechende Breite besitzt. v. $\mathrm{Br}$ a m an $\mathrm{n}$ bildete in No. 5 der mir gütigst zur Verfügung gestellten Fälle einell Hautlappen von den Dimensionen 8-18: $34 \mathrm{~cm}$, und das Resultat war ein vortreffliches. Die Breite der Basis des Lappens wird in entsprechendem Verhältnis zu seiner Länge zu bilden sein. Hier empfiehlt es sich, nicht zu sparsam zu sein, um eine genïgende Blutzufuhr zum Lappen $\mathrm{zu}$ ermöglichen.

Für die Bildung und Lösung des Lappens stellte Ma a s als Regel auf: der zul bildende Hautlappen ist möglichst in (ler Richtung des Gefässverlaufs herauszuschueiden, selbst wenn dadurch bei der Anheftung an den Defekt eine stärkere Drehung um seinen Stiel nötig sein sollte. Die Wahl der Stellung zum Mutterboden ist also bedingt durch den Gefässverlauf.

Diese Vorsichtsmassregel ist aber nicht unbedingt nötig, denn einmal sind die Hautgefässe sehr zahlreich und reich an Anastomosen, sodass ein grösseres Hautstück nicht von den Zweigen nur eines grösseren Gefässes gespeist wird. Andererseits zeichnen sich gerade die Gefässe der Haut der Extremitäten durch einen atypischen Verlauf bei den einzelnen Individuen aus, so dass schon hierdurch eine genaue Befolgung der Maas'schen Regel mit Schwierigkeiten verknïpft ist. von Bramann bildete in No. 1 der am Schluss näler zu besprechenden, von ihm opcrirten Fälle, zur Dekkung eiues Fusssohlen- und Fersendefekts einen Lappen aus der anderen Wade, dessen Basis nach aussen lag, so dass also die Längsachse des Lappens nicht mit der Richtung des Gefässverlaufs im Sinne von M a s zusammenfiel. Die Anheilung erfolgte in derselben Zeit wie in anderen Fällen.

Wird der Stiel des Lappens einer zu starken Drehung oder durch ungenügende Fixation einer Zerrung, einem dauernden Drucke ausgesetzt, so wird die Blutzufuhr zum Lappen behindert und seine Anheilung dadurch verzögert oder unmöglich. 
Dieffenbach war es in melreren Fällen aufgefallen, dass wälırend der Operation eine starke Hyperaemie des Lappens eintrat, und derselbe eine cyanotische Farbe annahı. Er liess deswegen die Hautlappen nach ihrer Ablösung kräftig bluten, suchte auch durch kalte Umschläge und Blutegel einer Hyperaemie, der häufigsten Ursache des Absterbens des Lappens, vorzubeugen und empfahl zu gleichem Zwecke, in dem Stiele keinen Arterienhauptast zu erhalten. v. Langenbeck wies aber nach, dass nicht der arterielle Zufluss die Hyperaemie bewirke, sondern die zu starke Drehung des Lappens un den Stiel und der dadurch behinderte venöse Abfluss; es tritt also konsekutive ischämische Gangrän ein.

Bei genügender Breite des Stieles verträgt ein Hautlappen schon eine ziemliche Drehung und Dislokation, ohne gangränös zu werden. Ausserdem kann man auch dadurch eine stärkere Drehung vermeiden, dass man bei der Lappenbildung den Längsschnitt derjenigen Seite, nach welcher der Lappen gedreht werden soll, etwas länger anlegt, als den der anderen Seite. Selbstredend muss aber durch einen genügenden Fixationsverband jeảe nachträgliche Zerrung oder Dislokation des Lappenstiels vermieden werden.

In der Voraussetzung, dass ein Lappen, welcher durch 2 Briicken mit dem Mutterboden zusammenhängt, besser ernährt würde, hat man auch doppelstielige Lappen empfohlen. Billroth, Sawicki, von Hacker ${ }^{1}$ ) und Andere berichten von guten Erfolgen. Der Lappen wurde von seiner Unterlage derart abgelöst, dass er an seinen Breitseiten mit ihr zusammenhängen blieb. Die betr. Extremität wurde daranf zwischen Lappen und Unterlage hindurchgesteckt und dann ersterer mit seiner Wundfläche auf dem Defekte befestigt.

Die Trennung der Stiele, wovon suatter die Rede sein soll, geschah dann in zwei Zeiten.

1) V. Ritter von Hacker. Über den Ersatz grösserer Hautdefekte durch Transpl. frischer ein- und doppelstieliger Lappen aus entfernten Körperteilen. Archiv f. klin. Chir. 37, 1888. 
Bei der Loslösung vom Mutterboden wird der einstielige Lappen durch 2 Längsschnitte und einen verbindenden Querschnitt abgegrenzt und von der Fascie oder mit derselben von der Unterlage abpräpariert.

v. Bramann löst die Fascie mit ab, wenn der Lappen zur Deckung eines Substanzverlustes dienen soll, in welchem der Knoclien freiliegt; andernfalls wird die Fascie mit der Unterlage im Zusammenhang belassen.

Bevor der Lappen aber auf dem Defekt befestigt werden kann, hat man diesen dazu vorzubereiten. Zeigt der Substanzverlust eine frische Wundfläche, so kann man sich mit sorgfältiger Butstillung begnïgen; ist die Wundfäche mit Granulationen bedeckt, so sind diese mit einem Rasirmesser oder besser mit dem scharfen Löffel zu entfemen, wie Thiersch es angegeben hat.

Hat man es mit Hautdefekten $\mathrm{zu}$ thun, welche durch ein Gescliwür oder durch Narben veranlasst sind, so sind diese vor der Operation durcli Excision zu entfernen. Bei Unterschenkelgeschwüren ${ }^{1}$ ) mit cirkulärer Narbe, welche durch Druck auf die zuführenden Gefässe Ernährung und Granulationsentwicklung hindert, ist die Narbe bis zur Fascie zu umschneiden, etwa 2 Querfinger breit vom Defektrande entfernt, $ı \mathrm{~m}$ ein Absterben des Narbendefektrings zı verhindern. Man hat dann die Bildung besserer Granulationen abzuwarten, ehe man zur Transplantation selbst schreiten kann. Denn es ist eine Hauptbedingung fïr die Anheilung eines Lappens, dass an den Rändern des Defekts überall normale Haut vorhanden ist und die Wundfläche aus normalem, nicht infiltriertem Gewebe besteht.

Bei ausgedehnten Substanzverlusten an Hand und Fuss hat man, wewn Sehuenverletzungen vorliegen. die Sehnennaht, bei Spitzfussstellung durcl Narbenkontraktur am Fuss die Tenotomie der Achillessehne der Plastik voranzuschicken.

Nach diesen Vorbereitungen werden die Ränder des Defekts, welche mit dem freien Rande des Lappens in Ver-

1) v. Hos etig-Moorh of Chirurg. Operationslehre. 
einigung kommen sollen, angefrischt; etwa überschüssiges Fettgewebe wird am Rande des Lappens abgetragen, um seine Wundränder gleichmässig zu machen.

Jede Blutung ist sorgfältig durch Compression zu stillen, erst dann erfolgt die Adaption des Lappens und seine Befestigung durch Nähte. Die verschiedentlich empfohlenen Entspannungsnähte sind unnötig und zu verwerfen, da sie anämisierend wirken. Zur Naht bedient man sich der Seide, sie ist dem von Maas empfohlenen Catgut vorzuziehen, zumal die Nähte 14 Tage liegen bleiben miissen.

Zur Bedeckung des freibleibenden wunden Teils der Ernährungsbrücke, des unbedeckt gebliebenen Defektrestes sowie der neu geschaffenen Wundfläche empfahl Maas, Borsalbe, dick auf Gaze gestrichen, zu verwenden, um hierdurch den Lappen vor Austrocknungsnekrose und Infection $\mathrm{zu}$ schützen.

v. Bramann verwendet hierzu mit Erfolg Jodoformgaze, ein untedingt reinlicheres Verfahren.

Den durch die Bildung des Lappens geschaffenen Defekt wird man sofort durch Hautfasciennälite zu verkleinern suchen, ehe man den Jodoformverband anlegt.

v. Langenbeck warnte vor dem Hinüberleiten der Brïcke iiber unverletzte Stellen der Haut zur Wundfläche des Substanzverlustes, indem er annahm, dass hierdurch die Gefahr des brandigen Absterbens für den Hautlappen gesteigert würde. Diese Vorsicht ist unnötig, wie sich gezeigt hat. Die Ausfüllung bestehender Liicken mit Jodoformgaze sichert vollkommen einen normalen Verlauf der Anheilung.

Damit der Lappen der Defektfläche iiberall gut anliegt, ind eine stärkere Exsudatbildung zwischen beiden verhindert wird, hat man sodann einen leichten aseptischen Druckverband anzulegen.

Hierauf folgt einer der wichtigsten Operationsakte, der der Fixation des empfangenden Körperteils an den gebenden. 
Es ist ein unbestreitbares Verdienst von Maas, auf die Wichtigkeit der Fixation ganz besonlers hingewiesen zu haben. Wieviele Misserfolge mögen wol vorher durch eine ungenügende Aneinanderlagerung der betr. Körperteile und dadurch verursachte Alteration des Lappens veranlasst worden sein! Ich erwähne nur einen Fall von Wutzer, welcher selbst zugab, dass die Befestigung durclı ledeine Schnürstrümpfe, wie er sie versucht hatte, nicht genïgte und eine Anheilung verhindern musste.

Vor allem kommt es bei dem Fixationsverbande darauf an, dass die Aneinanderlagerung der Körperteile sich bequem bewerkstelligen lässt und für den Patienten auf die Dauer nicht mit $\mathrm{zu}$ grossen Unannehmlichkeiten verbunden ist. Denn durch einen zul frühzeitigen Verbandwechsel diirfte, anch unter Narkose, die Anheilung des Lappens immerhin beeinträchtigt werden.

Maas empfahl, für alle Fälle den Gypsverband zu benutzen, und zwal umgab er zunächst jeden der aneinander gelagerten Körperteile für sich nit einer Gypskapsel und heftete dann erst den Lappen auf dem Defekt an; zum Schluss vereinigte er beide Gyyskalıseln durch einen dritten Gypsverband mit einander.

Zur Befestigung der oberen Extremitäten an den Thorax bedient sich von Bramann nicht wie Mas des Gypsverbandes, sondern Heftpflasterstreifen und gestärkten Gazebinden. An den unteren Extremitäten ist aber ein Gypsverband unbedingt erforderlich, da hier von dem Patienten viel ausgedehntere Bewegungen bei ungenïgender Befestigung möglich sind.

Die Fixationsverbände werden, wenn zweckmässig angelegt, von den Patienten gut vertragen. Die Steifheit der Gelenke, welche durch die lange Aneinanderlagerung hervorgerufell wird, ist nach Abnahme des Verbandes bereits innerhalb von 24 Stunden meist völlig verschwunden.

Ich komme jetzt zur Besprechung der Zeit der Stieldurchtrennung, welche eine besondere Wichtigkeit dadurch 
beansprucht, dass sie natïrlich erst nach erfolgter Anheilung des Lappens vorgenommen werden kann.

Die indischen Ärzte sollen die Hautbrïcke nach 25 Tagen durchtrennt haben, Tagliacozza gab den 15.-20. Tag zur gänzlichen Ablösung des Lappens an; Gräfe berichtet sogar vol einer erfolgreichen Operation, bei welcher er am 6. Tage den Stiel durchschnitt.

Wie sich durch zahlreiche Fälle aus der Literatur nachweisen lässt, erfolgt die Lappenanheilung bei jüngeren Individuen bedeutend schneller als bei älteren Patienten und auch bei kleineren Kindern, ein Moment, welches bei der Durchtrennung des Stiels sehr in Betracht kommen kanı.

Wenn wir von doppelstieligen Lappen absehen, so kann man die Ernährungsbrïcke des Lappens einzeitig oder in 2 oder mehr Zeiten durchtrennen.

Ma s empfahl, die Ablösung des Lappens von seiner Grundfläche an 10.-14. Tage auf einmal vorzunehmen. In einigen Fällen hat er selbst jedoch bedeutend früher den Lappenstiel durchtrennt.

Einmal sah er sich genötigt, wegen der grossen Unruhe des Patienten, bereits am 7. Tage den Verband wechseln zu müssen. Er fand den Lappen gut angeheilt und durchschnitt seinen Stiel am selben Tage.

Ein anderer Fall betraf einen $5 \%$ jährigen Knaben; hier nahm Mas die Stieldurchtrennung schon am 6. Tage vor, wohl an dem frühesten Zeitpunkt, welcher in der Literatur bekannt ist.

In beiden Fällen lag es wohl an dem jugendlichen Alter der Patienten und dem vollständigen Fehlen einer Randgangrän des Wunddefektes, dass die Lappen in dieser kurzen Zeit gut angeheilt waren. In einem 3. Falle löste Ma a bei einem 53 jährigen Patienten bereits am 9. Tage den Lappen völlig ab, auch hier verlief die Heilung ohne Störung.

Im allgemeinen wird man, wie Maas vorschreibt, am 


\section{$-26-$}

10. - 14. Tage den Stiel und zwar auf einmal durchtrennen können.

Eine zwei- oder mehrzeitige Stiellösung wird danı angebracht sein, wenn der Lappen eine zu bedeutende Grösse gehabt hatte, in der angegebenen Zeit noch nicht völlig: angelıeilt oder eine partielle Nekrose desselben eingetreten sein sollte.

v. Hacker empfahl zur zweizeitigen Durchtrennung die Zeit vom 6.-8. und 14.-17. Tage. Bei doppelstieligen Lappen nahm er am 12. Tage die Trennung des ersten, am 18. Tage die des zweiten Stieles vor.

Beim Durchschneiden des Stieles blutet der Lappen ziemlich heftig, ein Zeichen der neu entwickelten Gefässbildung in demselben.

Man hat sodann die von neuem gesprossten Granulationen zu entfernen, die Wundränder anzufrischen und nach gestillter Blutung den Lappen völlig auf dem Defekt durch die Naht zu befestigen.

Sollten kleinere Randteile des Lappens nekrotisch geworden sein, so sind dieselben, falls Demarkation da ist, abzutragen, und die Wundränder können dann von wetem vernäht werden. Die ïurigbleibenden Defekte sind am zweckmässigsten durch Transplantationen nach Th i er s ch zu decken.

Über das Verhalten des Lappens selbst während der Operation und besonders nach der Stieldurchtrennung sind verschiedene Angaben gemacht worden. Um zunäclıst eine von Maas in einem Falle und auch von Andern beobachtete Epidermisabstossung nach gänzlicher Ablösung des Lappens zu erwähnen, so ist dieselbe wohl als ein Zeichen der unvollkommenen Anheilung zu betrachten, zumal in diesen Fällen der Stiel zu selır früher 7eit durchtrennt wurde.

In anderen Fällen, in denen die Stieldurchschneidıng erst nach 10 und mehr Tagen vorgenommen wurde, trat eine solche Abstossung der Epidermis nicht ein; selbstredend ist liermit nicht die ganz oberfläclliche, stets bei der Haut vorkommende Epithelabschuppung gemeint. 
Inbetreff der Temperatur und des Aussehens des transplantierten Lappens beobachtete Fabrici, dass derselbe sich unmittelbar nach Durchtrennung der Basis kiihler anfïhlte und blasser aussal, als die umgebende Hant. Nach wenigen Stunden hatte sich jedoch die normale Temperatur und Farbe wieder hergestellt. Man wird dies Verhalten also leicht durch die Alteration der vasomotorischen Nerven durch den Schnitt und die wenn auch nicht gefährliche Anämie in der ersten Zeit erklären können.

Maas wiederuı bemerkte in einem Falle eine Temperaturerhöhung und eive längere Zeit anhaltende rötere Färbung des Lappens, welche erst bei Gebrauch der Körperstelle (der Lappen war auf die Ferse und Sohle verpflanzt) verschwand.

Nacl der Anheilung iiberragt der Lappen durch sein reichliches Fettpolster und das Auftreten einer gewissen Wulstung gewöhnlich etwas das Niveau der umgebenden Haut. M a a s konnte in dem oben erwähnten Falle beobachten, dass bei fortgesetztem Gebrauche des Fusses sich bald die zuerst zarte Epidermis verdickte, die Wulstung allmählich abnahm, und nach einiger Zeit das Aussehen und die Beschaffenheit der transplantierten und der benachbarten Haut keine Verschiedenheiten mehr zeigte.

Gleiches war in einem ähnlichen, durch v. Bramann operirten Falle zu bemerken.

Was das Verhalten der Sensibilität in dem transplantierten Hautstücke anbetrifft, so zeigt sicl, sobald dasselbe von seinem Mutterboden abgetrennt wird und nur noch durch eine Hautbrücke mit ihm in Zusammenhang bleibt, auch schon die Tastempfindung darin vermindert. v. Langenbeck machte darauf aufmerksam, dass bei Anlegen der Nähte der Patient die Durchstechung der Ränder des transplantierten Lappens gar nicht oder sehr wenig, die Nähte durch die Hautbrücke dagegen sehr schmerzhaft empfindet. Es schwindet also die Sensibilität der Nerven von der Peripherie vach dem Centrum zu, das Leitungsvermögen nimmt in centripetaler Richtung ab. 
Nach der, wenn auch nicht völligen Abtrennung des Lappens treten in ihm Paraesthesien auf, Taubscin, Kriebeln, Formikation, verschwinden aber nach kurzer Zeit wieder. Sie sind wol als eine Folge der Durchschneidung oder cler unumgänglichen Zerrung der Nerven des I Lappens in der Brïcke anzusprechen.

In den von Maas operierten Fällen wurden gewöhnlich bereits am selben Tage Berührungen und Nadelstiche auf dem Lappen gut localisiert, ebenso Wärme und Kälte richtig unterschieden. Eine Täuschung schien bei den getroffenen Vorsichtsmassregeln ausgeschlossen.

In 2 Fällen war schon nach 8 resp. 10 T'agen die Sensibilität eine beinahe normale zu nennen, nach einigen Monaten verhielt sie sich stets völlig wie die anderer Hantstellen.

In 2 anderen Fällen jedoch erwies sich die Sensibilität längere Zeit hindurch erheblich herabgesetzt. Die Schuld daran wird wohl zum Teil del frühzeitigen Stieldurchtrennung (6. und 9. Tag), hauptsächlich aber dem Umstande zuzuschreiben sein, dass das überpflanzte Hautstück unmittelbar auf dem freiliegenden Knochen zur Auflagerung' kam. In diesen Fällen blieb die Sensibilität in den ersten 6 Wochen stark abgeschwächt und stellte sich erst sehr allmählich wieder her.

v. Bramann bemerkte in 2 älınlichen Fällen das gleiche.

Meine Erörterungen stïtzen sich auf die von Herrn Prof. Dr. v. Bramann operierten und mir gütigst zur Verfügung gestellten Fälle, welche ich jetzt folgen lasse:

Richard Windisch, 26 Jahre alt, Bahnarbeiter aus Weissenfels.

Diagnose: Grosse Quetschwunde an der rechten Fusssohle mit ausgedehnter Abtrennung der Haut.

Allamnese:

Pat. war beim Rangieren verunglückt. Er hatte eine 
Lokomotive abgehängt und wollte sich aus den Schienen entfernen, dabei strauchelte er, und die rückwärts falırende Lokonotive überfuhr seinen rechten Fuss. Er wurde am 24. 3. 1891 mit einem Verbande in die Klinik gebracht. Die Wunde war gereinigt und genäht. Die Nähte werden, um die Wunde ganz übersehen zu können, entfernt.

Status praesens.

Der rechte Fuss erscheint stark geschwollen und ist teilweise blau verfärbt. An der Plantarseite ist die Haut mit subcutanem Fettgewebe abgehoben. Grössere Gefüsse und Nerven sind nicht verletzt. An einzelnen Stellen fehlt nur die Epidermis. An den Zehen und auf der Dorsalscite ist die Verletzung geringer. Der Calcaneus ist fast völlig von Haut entblösst, dieselbe ist wie eine Kappe abgehoben und nur noch an der äusseren Seite, am malleolus externus mit der übrigen Haut des Fusses im Zusammenhange; oberflächliche Knochenschichten sind an der unteren Seite des calcaneus abgerissen. Die IVunde wird desinficiert und mit Jodoformgaze tamponiert. Trockner Verband, Schienung und Hochlagerung des rechten Beines.

Allmählich reinigt sich die Wunde. Die nekrotisch gewordene Hautkappe wird entfernt. In der planta pedis liegen die intakten Sehnen der flexores frei, weiter hinten der calcaneus.

J. Juli. Der Wunddefekt betrifft die ganze rechte Ferse, sie nach hinten und zu beiden Seiten umgreifend, hinten nimmt er die ganze Breite der Sohle ein und verschmälert sich allmählich gegen die Zehenballen hin, welche er nicht mehr erreicht.

Die Granulationen sehen gut und frisch aus.

Verband mit essigsaurer Thonerde.

Zur Deckung des bestehenden Substanzverlustes wird die Transplantation eines gestielten Lappens aus dem andern Unterschenkel beschlossen.

17. Juli. Unter Chloroformnarkose werden die Granulationen der Wundflïche mit dem scharfen Löffel abgekratzt, 
der äussere Wundrand, am malleolus externus, wird angefrischt.

Nach vollkommener Blutstillung wird aus der linken Wade ein Lappen von entspr. Gröss", die Basis nach aussen gelegen, gebildet. $\mathrm{Er}$ wird mit der Fascie von der Unterlage abpräpariert und sein freier Rand mit dem angefrischten äusseren Defektrande der Sohle durch Seidennähte vereinigt. Tamponade aller unbedeckten Wundflächen mit Jodoformgaze. Aseptischer Verband. Die Beine werden in ihrer Stellung durch Gypsverband fixiert.

Am folgenden Tage wird der Gypsverband am rechten Knie, wegen starken Druckschmerzes etwas eingeschnitten.

25. Juli. Fieberfreier Verlauf. Fixationsverband wird gut vertragen.

31. Juli. Der Verband wird abgenommen. Der Lappen ist gut angeheilt, nur an der Aussenseite der Hacke zeigt sich cine oberflächliche Nekrose. Der Lappenstiel wird auf einmal durchtrennt. An den freien Randpartien wird das reichliche Fettgewebe des Lappens abgetragen und derselbe mit den angefrischten Wundrändern des Defekts an der Sohle vernäht, nach Entfernung der daselbst gesprossten Granulationen durch den scharfen Löftel.

Der Defect auf der linken Wade wird durch Hautfasciennälıte verkleinert. An der Sohle besteht nach vorn plantarwärts eine kleine, ungedeckte Wundfläche. Verband mit Jodoformgaze.

14. August. Fieberfreier Verlauf. Der Lappen ist gut angeheilt, besitzt normale Temperatur unil Farbe, nur an der Aussenseite besteht die erwähnte Nekrose. Diese wird entfernt. die vorhandenen kleinen Defekte werden durch Thierscli'sche T'ransplantationen gedeckt, desgleichen an der linken Wade.

12. November Die in mehrfachen Sitzungen ïberpflanzten Läppchen sind sämtlich angeheilt.

4. Dezember Patient wird entlassen. 
Dieser Fall ist ungefähr derselbe, welchen $\mathrm{M}$ ass als zweiten in seinen "weitere Fälle von Plastik mit frischen, gestielten Lappen aus entfernten Körperteilen" beschreibt. Auch dort war ein beträchtlicher, durch ein Geschwür gebildeter Defekt an Sohle und Ferse vorhanden, der Calcaneus lag, von Periost bedeckt, frei. Wie schon oben von mir erwähnt wurde, zeigte dieser Fall, dass ein Lappen auch zur Anheilung gelangt, wenn er nicht in der Richtung des Gefrissverlaufs im Sinne von Maas herausgeschnitten ist. Für die geringe, oberflächliche Nekrose, welche sich am Lappenrande einstellte, kann man wol kaum die Schnittführung zur Bildung des Lappens verantwortlich machen. Wäre thatsächlich durch Zerschneiden von Gefässen eine behinderte Blutzufuhr eingetreten, so würde sich die Nekrose nicht auf einen so winzigen Teil des Lappens beschränkt haben.

Der Lappen wurde mit der Fascie losgelöst, da er zum Teil auf den entblössten Knoclien zu liegen kam.

Der Stiel wurde nach 14 Tagen auf einmal durchtreant, während Mass diesen Operationsakt in seinem angegebenen Falle bereits am 6 . Tage vornalum. In beiden Fällen zeigte sich der Lappen gut angeheilt. Temperatur und Farbe des Lappens blieben normal. Die Sensibilität war in beiden Fällen stark herabgesetzt, Nadelstiche wurden in den erstell 4 Wochen nur als Druck empfunden; erst nach 8 Wochen hatte sich die Sensibilität fast völlig wieder hergestellt.

(siehe hierzu S. 28.)

Als siclı unser Patient nach einigen Monaten wieder in der Klinik vorstellte, war das Resultat ein vortreffliches. Patient ging in seinen gewöhnlichen Stiefeln ohne Beschwerden, die Hacke war nur durch etwas Watte geschiitzt. Das iiberpflanzte Hautstück zeigte die Sensibilität wie die Haut der anderen Hacke und hatte ungefähr dasselbe Aussehen wie die übrige Sohlenhant angenommen.

II.

Oskar Müller, 14 Jahre alt, Müller aus Wählitz.

Diagn os e: Zermalmung der l. Hand und grosse Quctschwunde an der rechten: 
Anamnese: Patient geriet am 18. April in der Wassermiihle mit der linken Hand zwischen 2 Walzen und kam, als er die ergriffene Hand frei machen Wollte, auch mit der rechten ins Getriebe. Durch den Riss des Transmissionsriemens standen hierauf die Walzen. Etwa 8 Stunden nach dem Unfall kommt Pat. in die Klinik.

Status praesens: Die 1. Hand ist zum grössten Teile von Haut- und Weichteilen entblösst, so dass teils die Sehnen in ihren anfyerissenen Scheiden, teils besonders an den Phalangen die Knochen blossliegen. An der rechten Hand verlänft eine tiefe Risswunde mit gequetschten und zerfetzten Rändern von der Volarseite der IJJ. Plialanx des Mittelfingers nach unten zur vola, biegt um den Kleinfingerballen nach dem dorsum $\mathrm{um}$ und endigt hier über denı $\mathrm{V}$. Metacarpus. Die Sehnenscheide des Flexor III ist eröffnet, die Haut des IV. und V. Fingers bläulich verfärbt, die III. Phalanx des $1 V$. und V. Fingers frakturiert.

18. April. Zunächst wird die linke Hand der ausgedelinten Zermalınung wegen, dicht ïber dem Handgelenk amputiert. Rechterseits werden die Fetzen an den Räıderı und im Grunde der Wunde abgetragen, letztere wird gereinigt und mit Jodoformgaze tamponiert.

Beide Arme in Suspensionsverband.

12. Mai. An der rechten Hand werden nekrotische Hautfetzen entfernt, unter ihnen zeigt sich eine gut granulierende Wundfläche.

19. Mai. IV. und V. Finger der l'. Hand siod durch Demarkation im Gelenk zwischen I. und II. Phalanx völlig gelockert und werden hier abgetragen.

26. Mai. IV. nnd V. Finger der r. Hand werden exarticuliert. Aus der Gegend des 1. Rippenrandes wird ein Lappen gebildet, dessen Basis nach unten sieht. Er wird mit der Fascie abpräpariert, und sein freier Rand mit der Wundfläche am dor'sum des metarcarp. III vernäht, nachdem die Granulationen entfernt siud. Die Wunde auf dem Thorax durch Hautfascienuähte verkleinert. Tamponade der Wund- 
flächen mit Jodoformgaze. Fixation des Armes an den T'horax durch Heftpflastertouren und gestärkte Binden.

10. Juni. Der Verband wird abgenommen und, da der Lappen gut angeheilt ist, der Stiel auf einmal durchtrennt, der Lappen völlig angenäht.

24. Juni. Defekt auf dem Thorax wird durch Thier s cls'sche Transplant. mit Erfolg gedeckt.

1. August. Passive Bewegungen!

10. Oktober. Temperatur, Aussehen und Sensibilität des Lappens ist normal. Die Prüfung der Beweglichkeit der Hand ergiebt:

Der III. Finger der r. Hand steht in starker Beugungskontraktur. Beim Versuch, denselben zu strecken, zeigt sich die Beugesehne im proximalen Interphalangealgelenk mit der Haut verwachsen.

Der II. Finger lässt sich passiv völlig beugen und strecken. Aktir wird derselbe in metacarpophal. Gelenk vollkommen gebeugt, im prox. Interphal.-Gelenk bis zum r. Winkel, im distalen gar nicht.

l)er Daumen ist aktiv vollkommen beweglich.

14. Oktober. Unter Chloroformnarkose und Asepsis wird durch einen in der Volarseite des III. Fingers über das Interphalangealgelenk geführten Schnitt die hier sich befindliche Narbe gespalten. Die Sehne zeigt sich hier mit der Haut und dem Knochen durch Narbengewebe verwachsen. Bein Versuche, den Finger nach Lösung der Sehne zu strecken, reisst diese, narbig verändert, in der Höhe des distalen Interphalangealgelenks. Sehnennaht. Hautnaht. Der Finger wird, im metacarpophal. Gelenk gebeugt, auf Schiene fixiert.

21. Oktober. Fixation des Finger's in etwas gestreckterer Stellung auf einer Schiene.

24. Oktober. Der Finger wird völlig gestreckt verbunden. Mit den ïbrigen Fingern werden andauernd passive Bewegungen vorgenominen.

27. Oktober. Leichte pass. Bewegungen an dem III. 
Finger, bei denen die Sehne in der ganzen Ausdehnung der Wunde mitgcht.

14. Dezember. Resultat: Der Daumen der rechten Hand ist frei und ziemlich kräftig beweglich. Der Zeigefinger ist im metacarpophal. Gelenk etwas schlotternd, in beiden Interphal. Gelenken beweglich, aktiv im ersten bis zum r. Winkel, im zweiten stumpfwinklich beugbar. Der 3. Finger ist in beiden Interph. Gelenken passiv nicht ganz streckbar. Bengung ebendaselbst beschrünkt; aktiv wird der Finger fast nur im metacarpophal. Gelenk. gebeugt.

Säntliche Wunden sind gut vernarbt. Die Gebrauchsfühigkeit der Hand beträot etwa 10\% der normalen.

Die Anlagerung der $r$. Hand an den linken Rippenrand war hier bequem zu bewerkstelligen, der Heftpflaster- und Gazeverband genügten völlig zur Fixation; der Fixationsverband selbst wurde von dem jungen Patienten ohne Beschwerden ertragen. Die Hand lag in dem z. Teil abgelösten Lappeu wie in einer Tasche, und es war von Anfang an bereits möglich, den grössten Teil des Lappens auf dem Defekte zu befestigen. Da sich der Lappen nach 14 Tagen gut angeheilt zeigte, wurde zu dieser Zeit der Stiel auf einmal durchtrennt.

In diesem Falle war der Erfolg der Plastik ein hochbedeutender, indem dem Patienten durch die Operation eine brauchbare Extremität erhalten wurde, hier um so wesentlicher, als die andere Hand vor der Amputation nicht gerettet werden konnte.

Nachdem die hinderlichen Stümpfe des IV. und V. Finger's entfernt waren, blieben dem Patienten noch Daumen, Zeige- und Mittelfinger, welche zum Zufassen, besonders auch zu feineren Verrichtungen, z. B. zum Schreiben, völlig genügen. Die Erwerbsfahigkeit des Patienten wurde also, wenigstens zum Teile wieder hergestellt. Allerdings war es hier noch nötig, durch eine zweite Operation die Gebranchsfähigkeit der Hand zu erhöhen; aber schon durch die Plastik erhielten die freiliegenden Sehnen eine schützende Decke, 
ohne welche sehr beträchtliche narbige Schrumpfungen entstanden wären, und Behinderung fast aller Bewegungell. Durch längere Zcit hindurch geiibte passive Bewegungen, besserte sich die Beweglichkeit und Brauchbarkeit der Finger noch weiter.

Der Lappen hatte das Aussehen der anderen Haut der Hand angenommen, war auf der Tnterlage verschieblich, seine Sensibilität normal.

\section{III.}

Ernst Ebert, Knabe aus Neustadt i. Th. 12 Jahre alt. Diagnose: Narbenkontraktur an der rechten Hand. rec. 9.6.1891; entl.: 12.10.1891.

Anamnese: Der Vater des Patienten besitzt oine Streichholzfabrik, worin sich Patient in Alter von 2 Jahren den rechten Unterarm und die rechte $H$ and dadurch verbrannte, dass er den im Wasser liegenden Stangenphosphor ergriff.

Anı stärksten wurde der Daumen und der IV. Finger verletzt, letzterer verlor damals ein Glied. Der Patient befand sich darauf 9 Monate lang in ärztlicher Behandlung.

Status praesens: An der Volarseite der rechten Hand zieht vom Daumen über das Handgelenk fort ein derber Narbenstrang, durch welchen die völlige Streckung des Handgelenks und die Daumenbewegung behindert wird. Der Daumen steht im rechten Winkel zum Unterarm und zum Handteller, er kann aus dieser Stellung kräftig adduciert werden; andere Bewegungen sind nicht ausfïhrbar. Der II. und III. Finger sind in der Beugung frei, in der Streckung leicht behindert. Vom IV. Finger existiert ein gut beweglicher aus $1^{1 / 2}-2$ Plialangen gebildeter Stumpf. Der V. Finger ist in Beugung und Streckung frei; der III. und V. convrrgieren iiber dem Stumpf des IV. mit einander.

\section{Sensibilität normal.}

30 Juni. Operation; Untel Chloroformnarkose wird die Narbe excidiert, worauf der Daumen etwas stärker abduciert 
werden kann; anscheinend sind jedoch im Carpo-metacarpalGelenke des Daumens schwere Folgeveränderungen ausgebildet, welche eine freie Beweglichkeit unmöglich machen. Schiene auf dem dorsum.

10. Juli. Die Stellung ist nur wenig gebessert, die Wunde hat sich mit frischen Granulationen bedeckt.

12. August. Operation: Die Wundränder der granulierenden Wunden werden angefrischt, die Granulationen mit dem scharfen Löffel abgekratzt.

Aus der linken Thoraxseite wird ein gestielter Lappen von entsprechenden Dimensionen gebildet. Seine 3 freien Ränder werden in der vola und an der ulnaren Seite des Defekts mit den Wundrändern desselben durch Naht befestigt, die Lappenbasis sieht nach unten.

Der Defekt auf der Brust wird durch Hautfasciennähte verkleinert. Auf die Wundflächen Jodoformgaze. Rechte Hand und Arm werden durch Heftpflastertouren am Thorax befestigt, dariber Verband mit gestärkten Gazebinden.

16. August. Der Lappen ist vollkommen erhalten und angeheilt. Die Hälfte der Basis wird durchschnitten.

Der Defekt auf der Brust wird durch Transplantationen nach Thierech gedeckt.

5. Sept. Der Hautlappen zeigt sich in ganzer Ausdehnung fest angeheilt, die Thiersch'schen Streifen nur zum Teil. Der Rest der Lappenbasis wird durchtrennt, die Lappenränder völlig angenäht.

Am Rande befinden sich noch einige kleine Wunddefekte;

Passive Bewegungen.

11. Okt. Der rechte Daumen erscheint etwas kürzel als der linke, vollkommen ist die Flexion nicht möglich. Adduktion, Abduktion und Opposition sind frei. An der Stelle des IV. Fingers befindet sich ein Stumpf, gebildet aus einer Juplikatur der beiden Grundphalangen. Die andern Finger sind frei und kräftig beweglich, ebensu das Handgelenk.

12. Okt. Patient wird entlassen. 
Dieser Fall zeigt, dass eine bedeutende Plastik auch bei narbigen Contrakturen möglich ist. Die beträchtlichen funktionellen Störungen im Handgelenk und im metacarpophalangeal. Gelenk des Daumens, besonders bei Streckversuchen, wurden durch die Operation ganz bedeutend gebessert, so dass dem Pat. jedenfalls eine brauchbare Hand geschaffen wurde.

Die Stieldurchtrennung erfolgte hier in 2 Zeiten, 14 T'age nach der Operation wurde etwa die Hälfte der Basis, 21 Tage nach der Operation der Rest des Stieles durchschnitten. Die Anheilung des Lappens war eine vollkommene.

Als sich Patient nach einigen Monaten wieder vorstellte, erwies sich die Sensibilität im transplantierten Lappen als normal, sein Aussehen war von dem der umgebenden Haut kaum verschieden. Die Beweglichkeit der Gelenke hatte sich seit Entlassung des Patient noch gebessert.

\section{IV.}

Hugo Brandt, 5 Jahre als, Bergmannssohn aus Pölsfeld. rec.: 17. XI. 1892, entl.: 19. II. 1893.

Diagnose: Quetschwunde der rechten Hand.

Anamnese: Der Knabe ist mit Ausnahme einer Vorbreunung, die er sich vor einem Jahre zuzog, und welche die Bengeseite des rechten Unterarmes betraf, immer gesund gewesen. Am 17. XI. 92 Mittags 12 Uhr kan er mit der rechten Hand in eine Dreschmaschine. Die Blutung soll nicht stark gewesen sein. Der Knabe ging selbst zum Dorfbach und wusch sich, erhielt von dem hinzugerufenen Arzte 3 Uhr Nachmittags einen Notverband und wurde nach Halle geschickt, wo er am Abend in der shirurg. Klinik aufgenommen wurde.

Status praesens: Auf dem dorsum der rechten Hand, vom Ende der ulna und des radius bis zu den Phalangen der Finger, fehlt die Haut fast vollständig, nur einzelne kleinere Lappen, an den Rändern stark gequetscht, sind noch vorhanden. Das Handgelenk selbst ist breit eröffnet und erscheiut auf der Ulnarscite stark gequetscht, das os multangulum minus ist zertrümmert, das os naviculare aus seiner 
Verbindung herausgerissen. Über das Handgelenk ziehen als blutig gefärbte, zum Teil zerrissene Stränge, an den Enden gleichfalls gequetscht, die Selhnen, von denen die des extensor pollicis und des ext. dig. quinti ganz erhalten, alle andern aber durchgerissen sind. - .

- Die beschädigten Sehnenstümpfe werden abgetragen; Sehmennaht. Das os multangulum minus und das os naviculare werden entfernt, das Gelenk drainiert. Antiseptischer Verband.

22. Nov. Zur Deckung des Substanzverlustes wird eine Lappenplastik beschlossen und zwar vom Thorax her, da der Haut- und Weichteildefekt zu ausgedehnt ist, um aus demselben oder dem andern Arme gedeckt werden zu können.

An der Bengeseite des rechten Arms zeigten sich ausserdem von frülher narbige Hautveränderungen.

Aus der linken Seite des Thorax wird ein grosser Lappen genommen, der nach unten sieht.

Seine freien Ränder werden mit den angefriscliten Wundrändern zum Teil vernäht. Die Wundflächen werden mit Jodoformgaze tamponiert, desgleichen das Handgelenk. Verband und Fixation des Armes am Thorax. Der neugeschaffene Defekt wird durch Hautfasciennälite verkleinert.

5. Dezember. Verbandwechsel. Der transplantierte Lappen zeigt auf dem dritten T'il seiner Oberfläche eine geringe Nekrose. Mit Durchschneidung des Stieles wird noch gewartet. Einige Nähte werden entfcrnt. Neuer Fixationsverband.

12. Dezember. Die oberflächlichen nekrotischen Partion des Lappens werden entfernt. Von der Nekrose sind die tieferen Schichten, das subcutane Fettgewebe verschont geblieben, so dass man hoffen darf, die Funktion der Sehnen erhalten zu können.

Der Lappenstiel wird zur Hälfte durchtrennt. Neuer Fixationsverband.

19. Dezember. Verbandwechsel. Der Lappen wird vollständig an der Basis abgetrennt, zeigt gute Vaskulari- 
sation und blutet stark. Er wird völlig durch Naht befestigt.

Suspensionsverband.

3. Januar 1893. Verband auf dem Thorax wird entfernt und durch einen Salbenverband ersetzt.

4. Januar. Verband der Hand wird abgenommen. Der Hautlappen zeigt sich gut angeheilt. Die Finger sind in den Phalangealgelenken selır gut beweglich. Es wird ein Verband mit essigsaurer Thonerde angelegt, welcher von jetzt ab täglich gewechselt wird, um die Hand in Seifenwasser baden zu können.

Die Metacarpophalangealgelenke können aktiv noch nicht gebraucht werden; passiv sind sie in sehr mässigem Grade beweglich. Besonders beschränkt dabei ist die Beweglichkeit des Mittelfingers.

17. Januar. Seit dem 4. Januar wird die Hand täglich in Seifenwasser gebadet und im Laufe des Tages dorsalflektiert gehaltan.

Die Bewegungen in den Meta:arpophalangealgelenken sind infolge der täglich vorgenommenen Dehnungen freier geworden, die Beweglichkeit des Mittelfingers hat sich jedoch wenig gebessert.

5. Februar. Plötzlich holies Ansteigen der Temperatur nach bisher fieberfreiem verlauf. Verbandwechsel. An der kranken Hand zeigt sich ein Erysipel. Stichelungen auf dem Unterarm, Verband init essigsaurer Thonerde. Am Avend nochmaliger Verbandwechsel. Die Hand ist stark gerötet und schmerzhaft. Verband mit Sublimat und Suspension der Hand.

6. Februar. Das Fieber hat etwas nachgelassen. Das Erysipel hat die gesetzten Grenzen nicht überschritten. Schmerzhaftigkeit und Rötung sind geschwunden. Verband mit essigsaurer Thonerde. Suspension.

8. Februar. Temperaturanstieg. Das Erysinel hat die Grenzen num doch ïberschritten Skarifikationen am oberen drittel des Oberarms. 
11. Februar. Temperatur abgefallen. Rötung und Schmerzhaftigkeit fehlen. Kein Fortschreiten des Erysipels.

15. Februar. Das Erysipel ist völlig versclıwunden. Die Wunde an der Hand ist völlig zugeheilt. Ein Verband wird als unnötig fortgelassen. Passive Bewegungen!

19. Februar. Patient wird mit zienlich frei beweglicher Hand entlassen. Zu Hause sollen die passiven Bewegungen täglich mehrmals fortgesetzt werden.

6. März. Patient stellt sich in der Klinik wieder vor': Er vermag sämtliche Finger vollkommen aktiv zu strecken. Dic Bewegungen im Handgelenk sind in fast normalen Grenzen aktiv ausführbar.

In diesem Falle war der frische, ausgedehnte Substanzverlust auf dem, Insulten stark ausgesetzten dorsum der Hand, kompliciert durch Sehnenverletzungen und breite Elöffnung des Handgelenks.

Transplantationen nach Thiersch hätten bei diesem tiefgreifenden Defekte nicht zu einer Heilung geführt; die Indikation zu einer Amputation war gegeben.

Nach 14 Tagen zeigte der Lappen eine teilweise, wenn auch ganz oberflächliche Nekrose. Mit der Durchtrennung des Stieles wurde deshalb gewartet. 21 Tage nach der Operation wurde eine Hälfte des Stieles durclischnitten, nach weiteren 7 Tagen der Rest. Die starke Blutung wal' ein Zeichen dafür, dass sich die Gefässe im Hautlappen in ausgedehntem Masse erneut hatten.

Der grosse Frfolg der Operation lag hier darin, dass die entblössten Sehnen eine kräftige, widerstandsfähige Decke crhielten, welche an jener Körperstelle unbedingt von Nöten ist, und dass das funktionelle Resultat ein vortreffliches war.

Der Erfolg wurde durch das plötzlich auftretende Erysipel nicht in Frage gestellt; eine kräftige Therapie führte auch hier zur baldigen Heilung.

Mit passiven Bewegungen wurde hier, so früh als möglich begounen, und mit der Zeit hierdurch das Fndresultat noch wesentlich gebessert. 
$\mathrm{V}$.

Wilhelm Hünerbein, 30 Jahre, Heizer ans Meisdorf. rec. 27. I. 91, entl. 4. VII. 91.

Diagnose: Weichteilverletzung am linken Vorderarm.

Anamnese: Pat. wurde am 27. I. 91 in einer Mühle dadurch verletzt, dass der Treibriemen eines Schwungrades den linken Arm erfasste und in grosser Ausdehnung die Haut vom Arme losriss.

Status praesens: Grosser, kräftiger Mann. Am linken Arme fehlt die Haut von der Mitte der Streckseite des Oberarms bis nahe zur Hand vollständio, in einer Länge von $30-33 \mathrm{~cm}$ und einer Breite von $6-10 \mathrm{~cm}$, letztere in Bereich des Ellenbogen. An der Bengeseite ist die Hautdecke zusammenhängend erhalten. Die Muskulatur ist an manchen Stellen zertrümmert; der musc. flexor carpi ulnar. und der flexor sublimis sind stark zerfetzt, dic corticalis des oberen Drittels der Ulna ist an ihrer hinteren Fläche fortgerissen. In der Gegend des metacarp. V sind die Weichteile gleichfalls verletzt, ebenso zwischen II. und III., sowie IV. und V. Metacarpus. Antiseptische Reinigung der Wunde. Tamponade aller Wundtaschen mit Godoformgaze.

5. Febrilar. Die ganze Wunde beginnt zu granulieren.

20. Februar. Iie Wunde zeigt gute Granulationen; kleine Fasciennekrose.

28. Februar. Die Wunde ist mit frischen, gut anssehenden Granulationen bedeckt; ein kleiner Teil des freiliegenden radius wird nekrotisch.

30. April. Operation: Nach Entfernung der Granılationen wird der linken Thoraxseite ein gestielter, c. $34 \mathrm{~cm}$ langer und an dem einen Ende 8, am andern c. $13 \mathrm{~cm}$ breiter Hautlappen entnommen und über dem Defekt des an den Thorax gelegten Armes durch Naht fixiert. Jodoformgaze auf die Wundflächen. Heftpflasterverband.

14. Mai. Verbandwechsel. Der Lappen ist gut angeheilt. Die Hälfte der Basis wird durchtrennt.

21. Mai. Der Rest des Stiels wird durchnitten. Naht. 4. Juli. Fieberfreier Verlauf. Patient wird entlassen. 
In diesem Falle, in welchem die Grösse des transplan. tierten Lappens, welcher zur Deckung des ungeheuren Hantund Weichteildefektes dienen sollte, besondere Erwähnung verdient, wurde gleichfalls ein vorzügliches Resultat erzielt. Patient war nach 4 Wochen Schonung zu Hause, wieder vollkommen im Stande, mit dem betr. Arme seine gewiss nicht leichte Arbeit als Heizer zu verrichten. Die Bewegungen im Ellenbogengelenk waren völlig normal und in kräftiger Weise ausführbar. Bei der Streckung des Armes krïmmen sich die Finger. Der Defekt auf der Brust ist gut verheilt.

Patienten wurde also cine notwendige Extremität durch die Operation erhalten und seine Erwerbsfähigkeit fast bis zur Norm wieder hergestellt.

Fassen wir zum Schlusse nun nochmals die Vorteile, welche durch solche plastische Operationen erzielt werden, und welche dieselben vor andern Transplantationsmethoden voraushaben, kurz zusammen, so erhalten wir folgendes Resultat:

Grosse, gestielte Hautlappen schrumpfen nach ihrer Anheilung nicht. Sie behalten ihre Elastizität vollkommen bei und sind auf der Unterlage wie normale Haut frei verschieblich.

Zahlreiche, treffliche Resultate haben gezeigt, dass sich die Lappen bei der Vernarbung sogar dehnten. In einem Falle, welcher von Maas operiert war und einen Defekt auf der patella betraf, verlängerte sich der transplantierte Hautlappen bei Beugung des Knies um $3,5 \mathrm{~cm}$ !

Vor allem geben diese Lappen eine ansreichende Bedeckung des Defektes ab. Lagen vorher durch das Trauma die Sehnen, Gefässe, Nerven oder Knuchen frei, so ist nach der Plastik durch die Dicke des Lappens eine kräftige Scliutzdecke vor Insulten geschaffen und eine adhärente Narbe vermieden, während andere Methoden in diesen Fällen dauernde Heilung nicht zu erzielen vermögen, da die neugeschaffene, dünne Epidermissschicht einen genügenden Schutz nicht abgiebt. 
Durch die plastische Bedeckung werden Ernährungsstörungen, welche durch lange Eiterung, chronische Geschwïre hervorgerufen sind, beseitigt; durch andere plastische Methoden sind solche geschwürige Prozesse einer dauernden Heilung nicht zugänglich.

Der hauptsächlichste aber und wichtigste Erfolg einer Plastik mit grossen, gestielten Hautlappen ist der, dass Funktionsstörungen verhindert oder beseitigt werden.

An den Gelenken lässt sich durch plastische Deckung die Bildung von narbigen Contrakturen verhüten; sind solche bereits vorhanden, so wird durch Narben-Excision und nachfolgende plastische Deckung die Beweglichkeit des betr. Gelenkes wieder hergestellt, und damit die Erwerbsfähigkeit des Patienten bedentend anfgebessert.

Vorher unbrauchbare Extremitäten lassen sich in brauchbare umwandeln; in vielen Fällen rettet sie eine Plastik vor der sonst unvermeidlichen Amputation.

M a as bemerkte mit Recht, dass auch die osteoplastische Resection des Calcaneus, welche von Mikulicz bei Hautgeschwüren und Defekten der Fersenhaut empfohlen wurde, sich durch eine plastische Deckung umgehen lässt; nur gleichzeitige Erkrankung des calcanens würde die Operation noch indiciren können.

An eine plastische Operation, welche wegen Funktionsstörungen an den Extremitäten vorgenommen wurde, hat sich zweckmässiger Weise eine regelrechte Massage und die fleissige Übung des betroffenen Gelenkes durch aktive und passive Bewegungen anzuschliessen; zu beginnen hat man damit je nach der Individualität des einzelnen Falles, natürlich erst nach völliger Anheilung der transplantierten Haut. Durch ein solchos Verfahren wird man mit der Zeit die Funktionsfähigkeit des Gelenkes und damit des ganzen Gliedes noch weiter bessern können. 
Zum Schlusse meiner Arbeit ist es mir eine angenehme Pflicht, meinem huchverehrten Lehrer, Herrn Prof. v. B r a m a n n, für gütige Übcrlassung des Materials, sowie liebenwïrdige Unterstützung bei Abfassung der Arbeit meinen besten Dank auszusprechen. 


\section{Literatur.}

Deutsche medizinische Wochenschrift.

Deutsche Chirurgie (Billroth u. Lücke).

Verhandlungen der deutschen Gesellschaft für Chirurgie.

Archiv f. klin. Chirurgie (v. Langen beck).

Centralblatt $f$. Chirurgie.

Eulenburg, Encycl. der ges. Heilkunde.

$\mathrm{K}$ önig. allgemeine Chirurgie.

Billroth, v. Winiwarter, allg. Chirurg. Therapie und Pathologie.

v. Mosetig-Moorhof, chir. Operationslehre.

v. Langenbeck, Vorlesungen über Akiurgie.

Zeis, Litteratur und Geschichte der plastischen Chirurgie.

Löbker, chir. Operationslehre.

Tillmanns, allg. Chirurgie.

I. Hahn, Transplant. ungestielter Hautlappen nach Wolfe mit Berücksichtigung der übr. Methoden (1888 Inaug,Diss. Kiel).

Prof. Dr. Haeser, Übersicht der Geschichte der Chirurgie und des chir. Standes.

Prof Dr.Thiersch, Über die feineren anatom. Veränderungen bei Aufheilung von Haut auf Granulationen (Leipzig 1874). Fabrici, Anaplastisches Verfahren zur Wiedererzeugung der Haut der inneren Handfläche. (1844).

Ernst Becker, Über Transplantation (Inauy.-Diss. Berlin 1881). 
O. Nagel, Über die Erfolge der Hauttransplantation nach Thiersch. (Aus der Tüb. Chir. K! des Herrn Prof. Bruns) 1889.

Dr. Hübscher, Beiträge zur Hautverpflanzung nach Thiersch. (Aus der Basel. chir. Kl. des Herrn Prof. Socin) 1889.

Wiener med. Wochenschrift.

Jungengel, Hauttranspl nach Thiersch. (Verh. der phys. med. Ges. Würzb Bd. XXV.)

Garré, Über die histologischen Vorgänge bei der Anheilung der Thiersch'schen Transpl. (Bruns, Beiträge zur klin. Chir. 1889. Sonderabdruck).

Poncet (Paris). Sur deux observations d'Autoplastie par la méthode italienre modifiée. (Bull. et num. de la soc. de chir. de Paris T. XIV, p. 29).

E. Plessing. (Mitteil, aus der chir. Klinik zu Leipzig). Hautverpflanzung nach Thiersch.

Deubel, Erysipèle gangréneux. Inoculation de la syphilis par l'intermédiaire des greffes. (Gaz. méd. de Paris 1881 No. 45).

M. Eisner. Über die Behandlungsweise des chron. Unterschenkelgeschwürs (1888). 


\section{Lebenslauf.}

Ich Georg, Richard Hartung, evangelischer Confession, wurde geboren am 16. Dezember 1867 zu Königsberg i. Pr. als Sohn des damaligen Buchdruckereibesitzers in Königsberg, jetzigen Rentiers in Leipzig Hermann Hartung. Ich besuchte zunächst in Leipzig die Teichmannsche Privatschule und dann das Nicolai-Gymnasiun, welches ich Ostern 1886 mit dem Zeugnis der Reife verliess, um Medizin zu studieren.

Ich besuchte sodann die Universitäten Heidelberg (S. S. 86. W. S. 86/87. S. S. 87) und Göttingen (W. S. 87/88 - S.S 89), wo ich am 8. Mai 1889 das tentamen physicum bestand. Im W. S. $89 / 90$ diente ich als Einjähriger beim kgl. preuss. magd. Jäg. Bat. No. 4 in Naumburg, studierte dann in Strassburg i. Els. (S. S. 90. W. S. 90/91) und Halle a. S. (S. S. 91 - S. S. 93). Durch die Güte der Herren Prof. Dr. v. Bramann und Geh. Med. - Rat Prof. Dr Weber war ich in der kgl. chir. Univ. Klinik vom 5. Dez. 1891 bis zum 1. April 1892, und in der kgl. med. Univ. Klinik vom 1. April 1892 bis zum 1. Okt. 1892 als Volontär beschäftigt. Am 4. August 1893 bestand ich das Examen rigorosum.

Während meiner bisherigen Studienzeit hatte ich die Ehre, folgende Herren Professoren und Dozenten zu hören:

Ackermann, Bernstein, v. Bramann, Bunge, von Bunsen, Damsch, Eberth, Eisler. Ebstein, Fischer, Gegenbaur, Harnack, v. Herff, v. Hippel, Hitzig, Kaltenbach, Krause, Kromayer, Kühne, Meissner, Merkel, v. Mering, V. Meyer, Oberst, P fitzer, Pott, Renk, Riecke, Schiefferdecker, Schirmer, Weber. 


\section{Thesen.}

I.

Bei ausgedehnten Haut- und Weichteildefekten an den Extremitäten ist die Transplantation grosser gestielter Hautlappen aus entfernten Körperteilen indiciert.

II.

In den Fällen, wo die vaginale Totalexstirpation wegen der Grösse des Uterus nicht auszuführen ist, ist die modificierte, ventrale Methode Freunds der sacralen Methode vorzuziehen.

III.

In allen Fällen von Sycosis parasitaria ist die Epilation vorzunehmen. 

COLUMBIA UNIVERSITY LIBRARIES (hsI, stx)
RD 121 H25 C. 1

Uber plastische Deckung von Hautdefekte

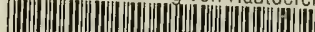

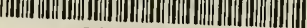

2002186497

\section{EOUNO}


